\title{
Desvendando a sexualidade de pessoas com sofrimento psíquico
}

RESUMO | Objetivo: Descrever aspectos referentes à sexualidade das pessoas com sofrimento psíquico atendidas em uma instituição de saúde mental no município do Rio de Janeiro. Métodos: Pesquisa qualitativa e descritiva. Participaram 12 usuários. Utilizaram-se entrevistas semiestruturadas para coleta de dados e análise de conteúdo. Resultados: Os participantes possuem vivências sexuais que refletem no seu comportamento social, como: abuso sexual, infecções sexualmente transmissíveis (IST), masturbação, entre outras. Conclusão: O tema sexualidade é tratado com superficialidade pelos profissionais de saúde; as pessoas com sofrimento psíquico precisam de educação em saúde sexual e reprodutiva para exercerem sua sexualidade de forma saudável e segura, melhorarem sua qualidade de vida e diminuir o estigma social que sofrem.

Palavras-chaves: Sexualidade; Enfermagem Psiquiátrica; Saúde Mental.

ABSTRACT | Objective: describe aspects related to the sexuality of people with psychic suffering attended at a mental health institution in the city of Rio de Janeiro. Methods: Qualitative and descriptive research. Twelve users participated. Semi-structured interviews were used for data collection and content analysis. Results: Participants have sexual experiences that reflect in their social behavior, such as sexual abuse, sexually transmitted infections (STIS), masturbation, among others. Conclusion: The topic sexuality is treated with superficiality by health professionals; People with psychic suffering need sexual and reproductive health education to exercise their sexuality in a healthy and safe way, improve their quality of life and reduce the social stigma they suffer.

Keywords: Sexuality; Psychiatric Nursing; Mental Health.

RESUMEN | Objetivo: describir aspectos relacionados con la sexualidad de las personas con sufrimiento psíquico atendidas en una institución de salud mental en la ciudad de Río de Janeiro. Métodos: Investigación cualitativa y descriptiva. Doce usuarios participaron. Se utilizaron entrevistas semiestructuradas para la recopilación de datos y el análisis de contenido. Resultados: Los participantes tienen experiencias sexuales que se reflejan en su comportamiento social, como el abuso sexual, las infecciones de transmisión sexual (ITS), la masturbación, entre otros. Conclusión: El tema de la sexualidad es tratado con superficialidad por profesionales de la salud; Las personas con sufrimiento psíquico necesitan educación sobre salud sexual y reproductiva para ejercer su sexualidad de manera saludable y segura, mejorar su calidad de vida y reducir el estigma social que sufren.

Descriptores: Sexualidad; Enfermería Psiquiátrica; Salud Mental.

\section{Izabella de Góes Maciel Tavares Campelo}

Mestre em Enfermagem. Unidade Integrada de Saúde Mental (UISM), Marinha do Brasil $(\mathrm{MB})$; e Escola de Enfermagem Anna Nery (EEAN), Universidade Federal do Rio de Janeiro (UFRJ).

\section{Bruna Gusmão de Matos Costa}

Mestre em Enfermagem (EEAN/UFRJ). Enfermeira. UISM/MB.

\section{Maria Angélica de Almeida Peres}

Mestre, Doutora (EEAN/UFRJ) e Pós-Doutora (Universidade Federal de Santa Catarina UFSC) em Enfermagem. Professora da EEAN/ UFRJ.

\section{Juliana Cabral da Silva Guimarães \\ Mestre em Enfermagem (EEAN/UFRJ).} Enfermeira. EEAN/UFRJ.

\section{Claudio Gruber Mann}

Doutor em Ciências / Saúde Pública (ENSP/ Fiocruz). Mestre em Enfermagem em Saúde Mental (EEAN/UFRJ). Especialista em Saúde Mental (IPUB/UFRJ) e Especialista em Gênero \& Sexualidade (IMS/UERJ). Pesquisador Colaborador da Columbia Global Centers - RJ e do Instituto de Psiquiatria da Universidade Federal do Rio de Janeiro (IPUB/UFRJ).

\section{Paulo Joaquim Pina Queirós}

Doutor em Dessarollo e Intervencion Psicologica (Universidad da Extremadura). Professor Coordenador da Escola Superior de Enfermagem de Coimbra.

Recebido em: 13/07/2019

Aprovado em: 16/07/2019
Introdução

1 sexualidade é um tema que vem ganhando destaque internacionalmente, sendo possível observar a discussão de aspectos relacionados à sua abordagem pelo profissional de saúde em estudos científicos atuais $^{(1,2)}$.

Estudos que relacionam Saúde Mental e Sexualidade têm demonstrado resultados resumidos a negligência, negação e controle medicamentoso, em detrimento de uma vivência saudável por parte das pessoas com transtornos psíquicos ${ }^{(2)}$.

Estigma, preconceito e negligência de direitos permeiam historicamente as vivências sexuais dessas pessoas, criando obstáculos para sua discussão, implicando no déficit de educação em saúde, contribuindo para casos de iniciação sexual em idade precoce - até 14 anos - gravidez indesejada, infecções sexualmente transmissíveis (IST) e comporta- 
mentos sexuais de risco. Esses preconceitos emergem também na abordagem dos profissionais de saúde, que pouco exploram as vivências sexuais das pessoas com sofrimento psíquico ${ }^{(2,3)}$.

A sexualidade é entendida como um aspecto central do ser humano ao longo da vida e inclui sexo, identidades e papéis de gênero, orientação sexual, erotismo, prazer, intimidade e reprodução. É experimentada e expressa em pensamentos, fantasias, desejos, crenças, atitudes, valores, comportamentos, práticas, papéis e relacionamentos. Frisa-se que nem todas essas dimensões sejam sempre experimentadas ou expressas. A sexualidade sofre, ainda, influência da interação de fatores biológicos, psicológicos, sociais, econômicos, políticos, culturais, éticos, legais, históricos, religiosos e espirituais ${ }^{(4)}$.

O exposto leva à reflexão de que, devido à interação de fatores biopsicossociais na determinação dos processos saúde-doença, os fatores subjetivos da pessoa com sofrimento psíquico - o que inclui a sexualidade - não podem ser desvinculados da assistência oferecida pelos profissionais de saúde mental.

Destarte, a sexualidade é parte inata da personalidade total, uma necessidade básica fundamental, devendo ser atendida de modo a permitir que o indivíduo tenha realização pessoal para que seja psicologicamente sadio ${ }^{(5)}$.

Atesta-se que há grandes problemas em relação ao reconhecimento da sexualidade das pessoas com sofrimento psíquico, reforçados pelo estigma e preconceito tanto entre profissionais de saúde quanto entre os próprios usuários, seus familiares e população em geral, prejudicando a atenção à saúde dessa população. Isso se expressa no uso propositado de medicamentos para controle da libido devido ao entendimento de que há um comportamento de risco ou de que não há a necessidade de uma vivência sexual por essas pessoas ${ }^{(2)}$.

Com o advento da Reforma Psiquiátrica Brasileira, um processo em cons-

66

Com o advento da Reforma Psiquiátrica Brasileira, um processo em construção, houve mudanças na assistência às pessoas com sofrimento psíquico, caracterizada pela luta por serviços substitutivos que favoreçam a construção da interação social, cidadania e autonomia, envolvendo aspectos culturais, sociais e econômicos ${ }^{(6)}$. trução, houve mudanças na assistência às pessoas com sofrimento psíquico, caracterizada pela luta por serviços substitutivos que favoreçam a construção da interação social, cidadania e autonomia, envolvendo aspectos culturais, sociais e econômicos $^{(6)}$. Nesta perspectiva, integra o hall da atenção à saúde da pessoa com sofrimento psíquico considerar o exercício da sua sexualidade e a experimentação de relações pessoais.

Mesmo almejando-se uma abordagem integral, os aspectos relacionados à sexualidade ainda são esquecidos na assistência à pessoa com sofrimento psíquico, refletindo em comportamento sexual de risco e com altas taxas de IST entre os usuários dos serviços de saúde mental ${ }^{(1)}$, explicitando a necessidade de desenvolver ações que visem à promoção da saúde e à prevenção de doenças, devendo, os profissionais de saúde, compreenderem a importância que o acesso à educação sobre uma vida sexual e reprodutiva saudável exerce na qualidade de vida dessas pessoas.

A pesquisa justifica-se por estudar a sexualidade da pessoa com sofrimento psíquico, alçando ferramentas para que os profissionais compreendam melhor as demandas destes usuários em todos os aspectos da sua subjetividade, possibilitando maior conhecimento da dimensão sexual, que requer atenção no cuidado.

A relevância da pesquisa está na aderência da temática com a "Agenda Nacional de Prioridades de Pesquisa em Saúde ${ }^{\prime(7)}$ e na contribuição científica que a pesquisa proporcionará, diante da predominância de estudos com abordagens atreladas a aspectos negativos e escassez de investigações acerca da vivência da sexualidade dessas pessoas ${ }^{(2)}$.

Diante do exposto, é objeto deste estudo os aspectos da sexualidade das pessoas com sofrimento psíquico atendidas em uma unidade de saúde mental no município do Rio de Janeiro. A questão norteadora foi: Qual a percepção das pessoas com sofrimento psíquico sobre sua sexualidade? O objetivo foi descre- 
ver aspectos referentes à sexualidade das pessoas com sofrimento psíquico atendidas em uma instituição de saúde mental.

\section{METODOLOGIA}

Pesquisa qualitativa, descritiva, justificada pela intencionalidade de estudar grupos sociais específicos no campo da saúde para qualificar o atendimento em saúde mental à população pesquisada ${ }^{(8)}$.

Foi cenário da pesquisa um Centro de Atenção Diária (CAD), anexo a um hospital psiquiátrico do município do Rio de Janeiro, funcionando como análogo aos Centros de Atenção Psicossocial (CAPS) - dispositivos de base comunitária, substitutivos aos hospitais psiquiátricos e que devem funcionar como porta de entrada das pessoas com transtorno mental no Sistema Único de Saúde - SUS ${ }^{(9)}$.

A escolha pelo cenário se deu pelo mesmo oferecer atendimento ambulatorial, acompanhamento clínico e atividades terapêuticas que objetivam estimular a convivência e treinar habilidades da vida prática e diária que são acometidas pelo sofrimento psíquico.

A equipe multidisciplinar do CAD, quando na coleta de dados, era composta por: uma psicóloga, uma enfermeira, uma médica psiquiatra, duas musicoterapeutas, três terapeutas ocupacionais, uma pedagoga, um educador físico e dez técnicos de enfermagem. Contava ainda com apoio semanal de mais uma psicóloga, uma assistente social e um médico clínico geral.

Esta pesquisa atendeu à Resolução n. ${ }^{\circ}$ 466/2012, do Conselho Nacional de Saúde (CNS) e, após a aprovação do projeto pelo Comitê de Ética em Pesquisa do Hospital Naval Marcílio Dias (CEP/ HNMD), sob o parecer consubstanciado n. ${ }^{\circ} 775.275$, foi iniciada a coleta de dados, realizada no período de setembro a outubro de 2016 através de entrevista semiestruturada. Os participantes assinaram o Termo de Consentimento Livre e Esclarecido (TCLE).

Os critérios de inclusão de partici- pantes foram: estar cadastrado no CAD e estar frequentando o serviço há, no mínimo, um mês. Agrega-se às atividades oferecidas pelo CAD a participação de usuários em regime de internação hospitalar, sendo definido como critério de exclusão estar internado, o que demandaria uma abordagem diferenciada em relação às pessoas em tratamento extra-hospitalar. Também foram excluídos os pacientes curatelados, devido à possível interferência no consentimento para participarem da pesquisa. Não houve distinção de sexo, orientação sexual e idade.

No momento da coleta de dados, o CAD contava com 42 inscritos e a delimitação do número de participantes para o estudo deu-se na medida em que houve a saturação dos dados, delimitando-se em 12 o número de entrevistados, tendo em vista que "uma amostra qualitativa ideal é a que reflete, em quantidade e intensidade, as múltiplas dimensões de determinado fenômeno e busca a qualidade das ações e das interações em todo o decorrer do processo" ${ }^{\prime(10)}$.

Foram participantes 6 homens e 6 mulheres que frequentavam as atividades propostas no projeto terapêutico do CAD, dos quais, de acordo com a Classificação Estatística Internacional de Doenças e Problemas Relacionados com a Saúde (CID-10), 10 possuíam diagnóstico de esquizofrenia (F20) e 2 de transtornos afetivo bipolar (F31). Em relação à idade, 7 usuários possuíam mais de 50 anos de idade, 3 entre 40 e 50 anos, 1 entre 30 e 40 anos e 1 menos de 20 anos.

As entrevistas foram transcritas e submetidas à leitura compreensiva, de modo a possibilitar a apreensão de seus conteúdos mais profundos. De posse dos dados coletados, revistos e selecionados, foi iniciado o processo de categorização, determinado pelas características significativas, fornecendo condições de manipular as complexas relações possíveis, oriundas dessa classificação ${ }^{(11)}$. Os participantes foram identificados no texto pela letra " $E$ " seguida do gênero $(F$, para gênero feminino e $M$ para gênero mascu- lino) e o número ordinal correspondente a ordem das entrevistas. A categorização considerou a semelhança dos temas surgidos no contexto dos discursos.

\section{RESULTADOS}

Optou-se pela descrição dos resultados seguindo-se a sequência lógica dada pelos participantes ao abordarem o tema em estudo, durante a coleta de dados.

A maioria dos participantes (8) declarou insatisfação com sua vida sexual, relacionada a causas diversas, como efeitos dos medicamentos em uso, dificuldade em encontrar um companheiro e impotência sexual:

"Isso me incomoda, porque não tem ninguém do meu lado" (EF6).

"Pra mim fica difícil por causa dos remédios e essas coisas [...] não dá pra arrumar ninguém" (EF10).

"Não porque ele é idoso [...] eu tô esperando porque ele teve AVC e tem impotência masculina [...] mas ele vai se tratar pra gente fazer amor [...] tô precisando ter mais sexo, mas meu companheiro ficou de se tratar, aí a gente vai viajar, passear [...] ele tá sempre doentinho, é hipertenso [...] mas ele prometeu pra mim que a gente vai se acertar no sexo" (EF8).

Outro ponto levantado com os participantes foi a vida sexual ativa, sendo perguntado há quanto tempo aproximadamente ocorreu a última relação sexual. Foi enfatizado pelo entrevistador que a prática de masturbação não assume o mesmo significado de vida sexual ativa, pois foi observado que alguns participantes fizeram esta associação em seus discursos. Os resultados mostram que 2 usuários nunca praticaram sexo e 10 não praticam, no mínimo, há 5 anos e 4 estão há aproximadamente 10 anos sem praticar.

Quanto à prática de masturbação, os 
participantes responderam sobre a frequência e o sentimento em relação ao ato: 5 usuários afirmaram praticar a masturbação e 7 negaram. Dos 5 participantes que relataram praticar a masturbação, 4 consideram a prática um ato normal/natural, prazeroso e que não está associada a pensamentos negativos, como podemos constatar nas suas falas:

"É uma coisa normal, me satisfaz" (EM3).

"Sinto prazer, prefiro me masturbar do que fazer sexo com qualquer um. Não me incomoda em nada, é uma coisa natural" (EF8).

Para um participante esta prática é diária e intensa, lhe causando desconforto, conforme podemos observar no relato abaixo:

"Sim, depende da ocasião, se eu tiver muito excitado, umas 4, 5 vezes por dia. É uma compulsão sabe, pra mim isso não é normal. Sexo deveria ser prazeroso, mas essa masturbação uma atrás da outra pra mim não é normal. Eu tenho vergonha [...] (de falar com o médico)" (EM5).

A não realização da masturbação foi justificada com motivo religioso por 2 participantes, cujos relatos estão a seguir:

"Eu não me masturbo, mas às vezes eu sinto vontade, mas como eu sei que é pecado, eu não me masturbo [...] é a mesma coisa que estar adulterando. Aí eu tomo um banho gelado e fico mais calma. Aí eu fico com dor de cabeça, passo mal [...]" (EF6)

"Não, porque eu sou crente, eu sou firme. [...] eu não tô pretendendo me desvirginar, porque é pecado, e só quando eu casar, e eu não tenho dinheiro, não te- nho um pé de meia pra fazer isso [...] então pra mim é melhor, né? Tá ajudando" (EF10).

Dentre os participantes, 3 informaram que não se masturbam porque apresentam diminuição do desejo sexual devido ao uso de determinados psicotrópicos:

"Tô tomando muito remédio de noite, eu não sinto (vontade). Quando eu pego no sono, eu não vejo mais nada, depois que eu tomo os remédios [...]" (EF10).

"Praticava, hoje em dia não mais, não funciona. Por causa do medicamento também. Não tenho prazer sexual nem para me masturbar" (EM4).

"Logo que eu me separei, eu vivia me masturbando, porque eu sentia falta [...] aí eu ficava me acariciando, me masturbando, sabe? Hoje em dia eu não tenho mais aquela vontade [...] não sei se é por causa dos remédios que eu tomo [...]" (EF7).

Quanto ao uso de preservativos, 3 informaram fazê-lo, sendo que 1 ressaltou que utiliza "às vezes", entretanto, há de se considerar na avaliação a predominância de uma vida sexual inativa entre os usuários.

Quando questionados sobre IST, 5 afirmaram já ter contraído alguma vez na vida. Destes, 4 responderam gonorréia, dentre os quais 1 relatou ter tido essa doença três vezes, o que remete a um comportamento sexual de risco quando na vida sexual ativa.

O abuso sexual foi relatado pela maioria dos participantes. 7 relataram já ter sofrido algum abuso sexual na vida, conforme explicitado nos relatos a seguir:

"Eu já [sofri abuso sexual], mas eu não entendi, eu achei que era pra fazer aquilo. Eu não estava gostando, mas eu não falava" (EF6).

"Sim, aos 10 anos. Eu trabaIhava na roça ainda. Os caras me cercaram, mas aí eu consegui fugir" (EM4).

"Com 11 anos começou, ele (pai) queria ver meus peitos [...] ficou só nisso [...] aí com 18 anos [...] fizemos sexo oral, tanto eu quanto ele [...] sexo oral... [...] Com 18 anos mesmo, fui pra rua e 8 homens me estupraram" (EF8).

"Já. Minha avó [...] ela começou [...] ela mostrou os seios [...] aí minha avó descobriu que eu tava a fim dessa garota, aí passou um tempo ela foi lá no shopping [...] aí eu falei: vó! Abracei a garota e falei: posso casar com ela? E minha avó disse: Não! Depois disso, minha avó mostrou os seios pra mim, começou a mostrar os seios" (EM11).

No que tange a abordagem da vida sexual pelos profissionais de saúde, 9 participantes negaram ter tido tal tipo de assistência, reforçando uma lacuna nos cuidados prestados pelos profissionais relacionados à vida sexual dos portadores das pessoas com sofrimento psíquico.

Dentre os participantes, 3 verbalizaram que gostariam que fossem desenvolvidas mais atividades educativas sobre sexo e DST nos serviços de saúde que frequentam e 2 deles utilizaram como exemplo palestras que já ocorreram na instituição-cenário:

"Aqui já teve palestra [...] gostaria que tivesse mais" (EF8).

"Acho que uma palestra é boa" (EM12).

Os resultados trazem importante reflexão para o cuidado psicossocial de pessoas com sofrimento psíquico, pois evidenciam características comuns referentes à sexualidade dos usuários que precisam ser discutidas no âmbito da 
atenção em saúde para que se pratique a clínica ampliada no atendimento de saúde mental, o que será discutido a seguir.

\section{DISCUSSÃO}

A partir do discurso dos usuários, identificaram-se alguns aspectos de sua sexualidade, como satisfação com a vida sexual, tempo decorrido desde a última relação sexual, masturbação, IST, abuso sexual e abordagem profissional sobre esta temática.

Evidenciou-se a insatisfação dos usuários do CAD com suas vidas sexuais, o que interfere na reabilitação psicossocial, que busca recuperar a autonomia dos sujeitos e garantir que tenha qualidade de vida na sociedade.

Não existe um conceito consensual sobre qualidade de vida, mas é patente que se relaciona com a subjetividade do indivíduo, abarcando o bem-estar, o grau com que as pessoas aproveitam as possibilidades de suas vidas e a percepção de satisfação com a vida, o que permite fazer a assertiva de que uma vida sexual satisfatória a integra ${ }^{(12)}$.

Notou-se que os participantes não são estimulados a falar sobre sexualidade no serviço de saúde, ficando este assunto de fora quando participam das atividades do CAD. Compete ao profissional de saúde refletir acerca da influência desta insatisfação na saúde mental do sujeito que já se encontra prejudicada em virtude da patologia psiquiátrica ou do estigma e preconceito que sofrem por serem usuários de serviço de saúde mental, podendo acarretar dificuldade em encontrarem parceiros sexuais ou em comportamentos sexuais de risco. Também é importante ampliar tal reflexão com o usuário, visando compreender sentimentos mobilizados e o planejamento de ações específicas para atender as demandas de cuidado nesta dimensão.

Nesse contexto, os resultados apontaram, também, um alto índice de abstinência sexual por mais de 5 anos, sendo que 2 usuários nunca praticaram sexo. Este resultado é significativo quando se tem em mente que a socialização dos sujeitos passa pela satisfação das necessidades humanas, como é a atividade sexual.

Existe o entendimento de que a sexualidade da pessoa com sofrimento psíquico é parte da psicopatologia, onde até manifestações mais singelas e rotineiras de expressão da sexualidade, como toque, beijo ou abraço, são vistas como psicopatológicas, incorporando o dispositivo da disciplina no cotidiano da assistência(13). Entretanto, a perda da libido e ausência de vida sexual também se fazem presentes, por exemplo, em um quadro depressivo ou na manifestação de sintomas negativos da esquizofrenia, onde há uma diminuição no interesse, na vontade e na capacidade de se relacionar afetivamente $^{(14)}$

As medicações antipsicóticas são frequentemente relacionadas a efeitos sexuais adversos. É comum, no exercício profissional, ouvir relatos de redução da libido e impotência sexual. De acordo com a National Schizophrenia Foundation, cerca de $39 \%$ das pessoas em uso dessas drogas referem queixa na esfera sexual. Em comparação com outros efeitos colaterais, como fadiga, ganho de peso e tremor, o efeito sobre a função sexual é considerado o mais problemático e importante razão de não aderência ao tratamento. Dentre as mulheres, os antidepressivos são os fármacos mais relacionados com disfunção sexual feminina(15).

A masturbação apareceu como uma atividade de realização sexual, remetendo a um sentimento positivo e prazeroso. É uma prática comum e saudável, que ajuda as pessoas a conhecerem melhor o próprio corpo e suas sensações de prazer. No entanto, comportamentos de manifestação da sexualidade, quando apresentados por pessoas com transtornos psíquicos, são entendidos como comportamentos demasiados complexos ou até problemáticos, ao contrário do que aconteceria caso estes fossem executados por pessoas ditas normais ${ }^{(16,17)}$.
Observa-se, na prática dos profissionais, a predominância de uma visão estigmatizante sobre o descontrole da sexualidade do usuário de serviços de saúde mental relacionado diretamente aos sintomas do transtorno ${ }^{(1)}$.

A masturbação compulsiva caracteriza uma adicção sexual, quadro psicopatológico em que o sujeito busca a realização do ato sexual de modo compulsivo, não importando as consequências a curto ou longo prazo ${ }^{(18)}$.

A masturbação com manipulação exagerada dos órgãos erógenos pode ocasionar lesões e até ferimentos graves. Nestes casos, indica-se que a masturbação seja detalhadamente orientada, demonstrada e treinada para evitar que o praticante se machuque com toques agressivos ou utilize objetos impróprios ${ }^{(19)}$.

Entende-se, desta forma, que a masturbação é uma expressão da sexualidade, entretanto, o sofrimento psíquico lhe confere um status patológico. Portanto, o cuidado em saúde não deve combatê-la, mas sim considerá-la como inerente àquela pessoa, atentando para orientar o indivíduo em casos de comportamentos compulsivos e/ou lesivos e até em casos de exposição pública de seu corpo.

As religiões sempre tiveram importante papel na formação do comportamento do ser humano, inclusive no sexual. Temas como adultério, homossexualidade, masturbação, virgindade, castidade, poligamia, aborto, casamento, divórcio, entre outros, sempre causaram controvérsia. A interpretação dessas passagens se diversifica de acordo com a vertente religiosa. Dentre os inúmeros atos de "luxúria", destacava-se a masturbação/onanismo, prática que se destinava unicamente à obtenção de prazer. Atualmente, a maioria das pastorais evangélicas tem a percepção de que práticas e manifestações sexuais como sexo oral, anal, acessórios, pornografia, adultério, masturbação, sonhos eróticos, prostituição, coito interrompido e homossexualidade são consideradas pecaminosas e doentias ${ }^{(20)}$. 
Apesar do número expressivo de participantes que não praticam relações sexuais, constatou-se um comportamento de risco durante a vida sexual ativa que contribui para o índice de IST, uma vez que, dos 12 entrevistados, 5 informaram que já tiveram alguma IST na vida, principalmente a gonorreia.

Independentemente da especificidade da psicopatologia, as ações de prevenção de IST merecem atenção especial, uma vez que as pessoas com sofrimento psíquico se encontram em situação de vulnerabilidade, seja pela própria psicopatologia ou por variáveis como características sociodemográficas, comportamentais e uso de substâncias ilícitas, o que contribui para a deterioração social e risco aumentado de IST, em comparação com outros grupos populacionais ${ }^{(21)}$.

Uma revisão bibliográfica contextualizando as questões do HIV/AIDS relacionadas à saúde mental apontou que as pessoas atendidas nos serviços de saúde mental pertencem a uma população com alto grau de vulnerabilidade para estas doenças, sendo comum, por parte destas pessoas, uma distorção da percepção com relação ao risco de infecção, dificuldades em adotar medidas preventivas e de buscar tratamento quando já infectados ${ }^{(22)}$.

Não discutir sexualidade com esses usuários implica na carência de ações de educação e prevenção de IST e HIV/AIDS, comprometendo o direito básico de todo indivíduo no Brasil, o direito à saúde ${ }^{(23)}$. Cabe aos profissionais de saúde a garantia deste direito, independente do tema a ser discutido e da população alvo.

Diante do exposto, a falta de conhecimento sobre a vida sexual dos usuários pode levar à negligência na avaliação de comportamentos de risco, na orientação de uma prática sexual mais protegida, bem como na proteção contra abusos sexuais.

A literatura aponta que a pessoa com sofrimento psíquico é mais vulnerável ao abuso sexual ${ }^{(1)}$. O presente estudo corrobora com essa assertiva, pois evidenciou que o abuso sexual foi vivenciado pela maioria dos participantes.
Diversos transtornos psíquicos têm sido relacionados a eventos traumáticos sofridos na infância, com níveis de gravidade que variam com o tipo de abuso sexual sofrido, sua duração e o grau de relacionamento da vítima com a figura que cometeu a agressão. Os traumas sofridos na infância são apontados como responsáveis por cerca de $50 \%$ das psicopatologias encontradas nos adultos, número bastante expressivo ao se considerar a gravidade do fato e o sofrimento que pode ser gerado quando o indivíduo é submetido a esta situação estressora ${ }^{(24)}$.

Em relação à abordagem profissional da sexualidade do usuário, constatou-se que ela se distancia do cuidado. O exercício da sexualidade dos usuários de serviços de saúde mental apenas é tratado quando o assunto é demandado pelo próprio usuário, diante de casos de violência sexual nas instituições ou de algum risco perceptível pelos profissionais de transmissão de IST/AIDS, havendo desconhecimento e falta de reflexão sobre o assunto entre os mesmos ${ }^{(1)}$.

Cada profissional de saúde deve se apropriar de seu próprio método para discutir o tema, ainda que haja resistência para tal, uma vez que a ideia de que a vida sexual desses sujeitos é uma extensão de seu transtorno psíquico ainda se faz presente. As concepções que os profissionais carregam consigo a respeito da sexualidade das pessoas com transtornos psíquicos influenciam diretamente na forma de ver e agir, remetendo a não valorização da vida sexual do usuário e sua sexualidade ${ }^{(5)}$.

Na prática, nota-se um despreparo dos profissionais de saúde em lidar com a sexualidade humana, onde se evidencia a atuação sob a perspectiva do modelo biológico, em detrimento da abordagem da sexualidade enquanto fenômeno social, cultural e subjetivo. Há invisibilidade e ocultamento da sexualidade da pessoa com sofrimento psíquico pelos profissionais de saúde, exceto quando a sexualidade é percebida nos momentos de crise, sendo relacionada a algo patológico.
Desta forma, as intervenções terapêuticas sobre a sexualidade não integram o cuidado devido às crenças, valores, preconceito e falta de conhecimento dos profissionais sobre a sexualidade humana ${ }^{(13)}$.

As atividades educativas agregam conhecimento para muitos portadores de transtornos psíquicos, devendo ser cada vez mais desenvolvidas com o intuito de estabelecer hábitos sexuais saudáveis. É importante ressaltar também que as atividades a serem desenvolvidas devem ser realizadas com temática definida, de interesse dos usuários e contextualizando a realidade da comunidade, uma vez que a reabilitação psicossocial é um dos principais objetivos da Reforma Psiquiátrica ${ }^{(25)}$.

\section{CONCLUSÃO}

Conclui-se que esta pesquisa atingiu o objetivo proposto, uma vez que trouxe dados sobre a sexualidade das pessoas com sofrimento psíquico e apontou a necessidade da equipe de saúde mental considerar no cuidado essa esfera da dimensão biopsicossocial dos usuários.

Evidenciou-se que a satisfação sexual, inserida na satisfação com a vida, estabelece um elo com a qualidade de vida. As falas apresentadas manifestaram a insatisfação com a vida sexual, bem como um alto índice de abstinência sexual. A "patologização" da sexualidade da pessoa com sofrimento psíquico aliada aos sintomas sexuais das psicopatologias e efeitos sexuais adversos dos psicofármacos também contribuem para esses resultados.

A realização sexual por meio da masturbação é prática saudável e exercida pela população em geral, sendo, também, observada nos entrevistados, mas constatou-se que, como integrante da sexualidade da pessoa com sofrimento psíquico, é estigmatizada. Entretanto, a prática compulsiva foi observada em apenas 1 usuário - essa sim merece ter um olhar terapêutico ao causar desconforto e acarretar possíveis danos. 
A vulnerabilidade da pessoa com sofrimento psíquico alinha-se ao comportamento de risco sexual, evidenciada na literatura e no presente estudo. Desconsiderar essas questões implica em omitir-se às necessidades de saúde desses usuários, o que alerta para a importância das ações educativas e preventivas de IST.

Reforçando a vulnerabilidade das pessoas com sofrimento psíquico, o abu- so sexual foi vivenciado pela maioria dos participantes e relaciona-se a psicopatologias. Sendo um fato grave e gerador de sofrimento, a equipe de saúde precisa trabalhar seu impacto junto ao usuário.

Por fim, esse estudo reforçou a distância existente entre a sexualidade e o cuidado das pessoas com sofrimento psíquico, indicando a relevância que essa dimensão assume na sua satisfação com a vida e socialização. As intervenções terapêuticas e atividades educativas devem ser fortalecidas para incentivar a adoção de hábitos sexuais mais saudáveis por essa população vulnerável, bem como trabalhar a diminuição do estigma e do preconceito, ainda existente, entre os profissionais, familiares e população em geral diante do exercício da sexualidade de pessoas com sofrimento psíquico.

\section{Referências}

1. Mann CG, Monteiro S. Sexualidade e prevenção das IST/aids no cuidado em saúde mental: 0 olhar e a prática de profissionais no Município do Rio de Janeiro, Brasil. Cad Saúde Pública [Internet]. 2018 [citado em 2018 jul. 5]; 34(7):e00081217. Disponivel em: http://www.scielo.br/scielo.php?pi$\mathrm{d}=$ S0102-311X2018000705009\&script=sci_abstract\&tlng=pt.

2. Detomini VC, Rasera EF, Peres RS. Sexualidade e saúde mental: vivências, serviços e estigmas. Revista da SPAGESP [Internet]. 2016 [citado em 2018 jul. 5];17(2): 81-95. Disponivel em: http://pepsic.bvsalud.org/scielo.php?script=sci_arttext\&pid=S1677-29702016000200007.

3. Spinola MCR, Béria JU, Schermann LB. Fatores associados à iniciação sexual em mães de 14 a 16 anos em Porto Alegre/RS, Brasil. Ciênc Saúde Coletiva [Internet]. 2017 [citado em 2018 jul. 5]; 22(11):3755-762. Disponível em: http://www.scielo.br/scielo.php?pid=S1413-81232017021103755\&script=sci_abstract\&tlng=pt.

4. World Health Organization. Developing sexual health programmes: a framework for action. Geneva: World Health Organization; 2010.

5. Townsend MC. Enfermagem Psiquiátrica: Conceitos de Cuidados. 3 ed. Rio de Janeiro: Granabara Koogan; 2011.

6. Costa JP, Jorge MSB, Coutinho MPL, Costa EC, Holanda ITA. A reforma psiquiátrica e seus desdobramentos: representações sociais dos profissionais e usuários da atenção psicossocial. Psi Saber Soc. [Internet]. 2016 [citado em 2018 jul. 5]; 5(1):35-45. Disponível em: https://www.e-publicacoes.uerj.br/index.php/psi-sabersocial/article/view/15855.

7. Ministério da Saúde. Secretaria de Ciência, Tecnologia e Insumos Estratégicos. Departamento de Ciência e Tecnologia. Agenda nacional de prioridades de pesquisa em saúde. 2 ed. Brasília, DF: Editora do Ministério da Saúde; 2008.

8. Minayo MCS. 0 desafio do conhecimento: pesquisa qualitativa em saúde. 12 ed. São Paulo: Hucitec; 2010.

9. Ministério da Saúde, Gabinete do Ministro (BR). Portaria n. ${ }^{\circ} 336$, de 19 de fevereiro de 2002. Dispõe sobre os Centros de Atenção Psicossocial - CAPS, para atendimento público em saúde mental. Brasília, DF: Diário Oficial da União; 2002.

10. Minayo MCS. Amostragem e saturação em pesquisa qualitativa: consensos e controvérsias. RPQ [Internet]. 2017 [citado em 2018 jul. 18]; 5(7):1-12. Disponível em: https://editora.sepq.org.br/index.php/rpq/article/view/82/59. 11. Marconi MA, Lakatos EM. Técnicas de pesquisa: planejamento e execução de pesquisas, amostragens e técnicas de pesquisa, elaboração, análise e interpretação de dados. 7 ed. São Paulo: Atlas; 2008.

12. Noronha DD, Martins AMEBL, Dias DS, Silveira MF, De Paula AMB, Haika DS. Qualidade de vida relacionada à saúde entre adultos e fatores associados: um estudo de base populacional. Ciênc Saúde Coletiva [Internet]. 2016 [citado em 2018 dez. 12]; 21(2):463-74. Disponível em: http://www.scielo.br/ scielo.php?pid=S1413-81232016000200463\&script=sci_abstract\&tlng=pt. 13. Ziliotto GC, Marcolan JF. Percepção de trabalhadores de enfermagem sobre sexualidade de portadores de transtornos mentais. Acta Paul Enferm [Internet]. 2013 [citado em 2018 dez. 12]; 26(1):86-92. Disponível em: http:// www.scielo.br/scielo.php?pid=S0103-21002013000100014\&script=sci_abstract\&tlng $=$ pt.
14. Elkis H, Freitas RR. In: Martins MA, Carrilho FJ, Alves VAF, Castilho EA, Cerri GG (Eds). Clínica Médica. 2 ed. Ampl. Ver., v.6. Doenças dos olhos, doenças dos ouvidos, nariz e garganta, neurologia, transtornos mentais. Barueri: Manole; 2016.

15. Cordás TA, Laranjeiras $M$. Efeitos colaterais dos psicofármacos na esfera sexual. Rev Psiquiatr Clín [Internet]. 2006 [citado em 2018 dez. 12]; 33(3):168-73. Disponível em: http://www.scielo.br/scielo.php?pi$\mathrm{d}=\$ 0101-60832006000300007 \&$ script=sci_abstract\&ting=pt.

16. Barros JV. A masturbação nos livros didáticos de ciências: uma análise a partir dos conceitos de biopolítica e de dispositivo da sexualidade. Revista Dialectos [Internet]. 2017 [citado em 2018 dez. 12]; 4(11):116-26. Disponível em: http://www.periodicos.ufc.br/dialectus/article/view/31004.

17. Maia ACB. Reflexões sobre a educação sexual da pessoa com deficiência. Rev Bras Educ Espec [Internet]. 2001 [citado em 2018 nov. 20]; 7(1):35-46. Disponível em: https://abpee.net/homepageabpee04_06/artigos_em_pdf/revista7numero1pdf/3bortolozzi_maia.pdf.

18. Padilha Netto NK, Cardoso MR. A adicção sexual nas fronteiras da perversão. Rev Latinoam Psicopatol Fundam [Internet]. 2017 [citado em 2018 nov. 20]; 20(4):705-27Disponivel em: http://www.scielo.br/scielo.php?script=sci_abstract\&pid=S1415-47142017000400705\&lng=en\&nrm=iso\&tlng=pt.

19. Facion JR. As Doenças Mentais na Infância e na Juventude. 0 Psicólogo e suas funções. Uma visão Humanística e Comportamental (A Sexualidade na Doença Mental). Anais do IV Encontro Paranaense de Psicologia; 1990; Curitiba. Conselho Regional de Psicologia do Paraná; 1990.

20. Duarte AJO. Religião e comportamento sexual: concepções cristãs sobre sexualidade. Relegens Thréskeia [Internet]. 2017 [citado em 2018 nov. 21]; 6(1):74-98. Disponível em:

21. https://revistas.ufpr.br/relegens/article/download/54134/34185.

22. Braz MV, Silva Filho N. Prevalência da infecção pelo HIV em pacientes psiquiátricos que fazem uso de um serviço público de saúde. Mudanças [Internet]. 2015 [citado em 2018 nov. 21]; 23(1):21-9. Disponível em: https://www. metodista.br/revistas/revistas-ims/index.php/MUD/article/view/5192/4985.

23. Carvalho CML, Braga VAB, Galvão MTG. Aids e saúde mental: revisão bibliográfica. DST - J Bras Doenças Sex Transm [Internet]. 2004 [citado em 2018 nov. 21]; 16(4): 50-5. Disponivel em: http://www.dst.uff.br/revista16-4-2004/9.pdf.

24. Barbosa JAG, Guimarães MDC, Freitas MIF. Sexualidade e vulnerabilidade social em face das infecções sexualmente transmissiveis em pessoas com transtornos mentais. Rev Méd Minas Gerais [Internet]. 2013 [citado em 2018 dez. 8]; 23(4). Disponível em: http://rmmg.org/artigo/detalhes/407. 25. Aded NLO, Dalcin BLGS, Moraes TM, Cavalcanti MT. Abuso sexual em crianças e adolescentes: revisão de 100 anos de literatura. Rev Psiquiatr Clín [Internet]. 2006 [citado em 2018 dez. 2]; 33(4):204-13. Disponível em: http://www. scielo.br/scielo.php?script=sci_arttext\&pid=S0101-60832006000400006. 26. Duarte EOS, Nasi C, Camatta MW, Schneider JF. Caracterização das práticas de assistência na rede de atenção em saúde mental: revisão integrativa. Rev Gaúcha Enferm [Internet]. 2012 [citado em 2018 dez. 2] 33(4):191-9. Disponivel em: http://www.scielo.br/scielo.php?script=sci_arttext\&pid=S1983-14472012000400024. 